\title{
A multi agent recommender system that utilises consumer reviews in its recommendations
}

\section{Debbie Zhang}

Faculty of IT,

University of Technology, Sydney,

P. Box 123 Broadway 2007, Australia

E-mail: debbiez@it.uts.edu.au

\section{Simeon Simoff*}

College of Health and Science, University of Western Sydney,

Locked Bag 1797, South Penrith DC, NSW 1797, Australia

E-mail: s.simoff@uws.edu.au

*Corresponding author

\section{Silvana Aciar}

University of Girona, Campus Montilivi, Building P4, 17071 Girona, Spain

E-mail: saciar@eia.udg.es

\section{John Debenham}

Faculty of IT,

University of Technology, Sydney,

P. Box 123 Broadway 2007, Australia

E-mail: debenham@it.uts.edu.au

\begin{abstract}
Consumer reviews, opinions and shared experiences in the use of a product form a powerful source of information about consumer preferences that can be used for making recommendations. A novel approach, which utilises this valuable information sources first time to create recommendations in recommender agents was recently developed by Aciar et al. (2007). This paper presents a general framework of this approach. The proposed approach is demonstrated using digital camera reviews as an example.
\end{abstract}

Keywords: recommender systems; multi-agent systems; text mining; ontology; intelligent agents.

Reference to this paper should be made as follows: Zhang, D., Simoff, S., Aciar, S. and Debenham, J. (2008) 'A multi agent recommender system that utilises consumer reviews in its recommendations', Int. J. Intelligent Information and Database Systems, Vol. 2, No. 1, pp.69-81. 
Biographical notes: Debbie Zhang is a research fellow in the e-Markets Research Group (e-markets.org.au) at the University of Technology, Sydney. Her work includes developing smart text-mining agents to extract unstructured text and numerical data from the internet and apply machine-learning techniques to verify, combine, filter, and condense the extracted information for e-negotiators. Her research interests include text mining, modelling of financial market behaviours, and discrete-element modelling. She received her $\mathrm{PhD}$ from the University of Queensland, Australia.

Simeon Simoff is a Professor of Information Technology and Head of School of Computing and Mathematics, University of Western Sydney, an Adjunct Professor, Faculty of IT at the University of Technology, Sydney and the leader of the e-Markets Research Group. His research interests include information-rich trading environments and technologies that facilitate extraction, synthesis, and delivery of condensed information from them, including network data mining, text analytics, multimedia, and visual data mining. He's also exploring the use of context networks that are established by and surround the electronic trading processes. He received his $\mathrm{PhD}$ in Computer Science from the Moscow Power Engineering Institute.

Silvana Aciar has a $\mathrm{PhD}$ in Information Technology from the University of Girona's Department of Electronics, Computer Science, and Automatic Control. She is also a member of the Agents Research Lab in the Institute of Informatics and Applications at the UdG. Her research interests include multiagent systems, recommender systems, and ontologies. She received her MSc in information technology from the UdG.

John Debenham is a Professor Emeritus in the Department of Software Engineering, Faculty of IT at the University of Technology, Sydney and a member of the university's E-Markets Research Group. His recent work has focused on multiagent systems, e-negotiation, and argumentation agents. He's particularly interested in the design of agents that can exploit the information-rich environment of electronic markets. He received his $\mathrm{PhD}$ in Computer Science from the University of Sydney.

\section{Introduction}

Review comments and informal evaluations are a powerful and expressive source of user preferences. Product review forums and discussion groups are popular ways for consumers to share their experiences with a product (Dellarocas, 2003, 2004; Curien et al., 2006). There is growing evidence that such forums inform and influence consumers' purchase decisions (Senecal and Nantel, 2004; Chevalier and Mayzlin, 2003). These reviews provide valuable source of information for recommender systems.

Although the importance and value of such information has been recognised, there are not many recommendation systems that utilise this information due to the problem of automating the incorporation of unstructured data in the recommendation process (Adomavicius, 2005). Consumer reviews are collected and preserved in free form text. Having all that wealth of text information, the problem for a recommender system is how to 'understand' the text information and make 'meaningful' recommendation based on these data. A recommender agent, which utilises review comments to create recommendations, was recently developed by Aciar et al. (2007). Our earlier research 
developed the overall approach and the methodologies that support it. The approach is illustrated in Figure 1. A selection and retrieval process of unstructured/semi-structured individual reviews, based on consumer level of expertise in using a product has been developed. In order for the recommendation process to utilise the textual information, an ontology was defined so the review comments could be represented in structured formats. Each individual comment is then mapped into the ontology as an instance. A prioritising mechanism for producing the recommendation results was developed. This paper is focused on the computational architecture of the recommendation system, realised as a multi agent structure. The design adopted the recently developed approach towards engineering multi-agent systems as electronic institutions (Sierra et al., 2004), which facilitates design and development of efficient and robust open agent organisations.

Figure 1 The approach for automatic incorporation of the impact of consumer opinions in the recommendation process

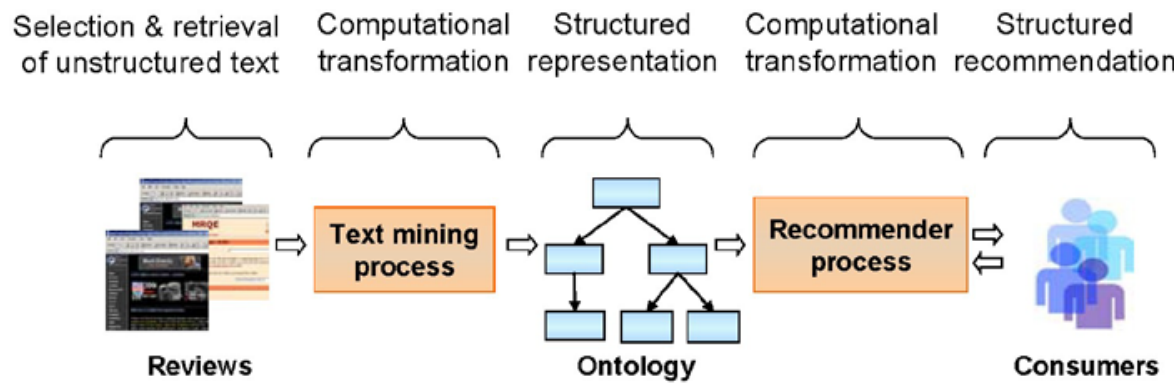

The rest of the paper is organised as follows: Section 2 provides a brief introduction to the recommender system, redefined as a multi-agent system. It includes three types of agents: web mining agent, recommendation agent and user interface agents. Sections 3-5 detail each type of agent respectively. The implementation issues are presented in Section 6 . Section 7 concludes the paper and provides directions for future research.

\section{Recommender system based on consumer review comments}

Consumer opinions are scattered across various websites, each with different layout. The recommender system automatically collects relevant comments from product reviews from such websites and generates recommendation from these comments. Figure 2 shows the multi agent interaction structure of the recommender system. This structure is then formalised in terms of the electronic institutions methodology.

According to the electronic institution methodology, we specify the roles that each agent can have. Each agent can play multiple roles, which are specified in terms of standardised patterns of behaviour. Further, we keep the term 'type of agent' to preserve the consistency of our presentation with the process description. The multi-agent structure of the recommender system relies on three types of agents: Web mining agent, Recommendation agent and User interface agent. The roles each agent plays are: 
- Web mining agent. Web mining agents are responsible for retrieving review comments from predefined websites. Each agent monitors one website and constantly delivers new information (review comments) to the Recommendation agent. It also cleans the extracted data for the Recommendation agent. The agent automatically detects changes in the website format and adjusts to those changes (the approach adapts our earlier work on automatic news retrieval for information mining discussed in Zhang and Simoff (2004)).

- Recommendation agent. It acts in two roles:

- $\quad$ text mining role in order to convert the comments from free text form to a structured ontology

- $\quad$ recommendation role, in order to calculate the rating of each product from the review comment ontology and formulate specific recommendations based on the rating calculations.

- User interface agent. User interface agents allow users to connect to the system via the internet. They depict users' requirements in terms of the advice they need and provide personalised assistance according to those needs.

One of the benefits that the electronic institutions methodology offers is off-the-shelf means for interactions between the agents. The protocol of agent communication is defined by the dialogic framework. The dialogic framework establishes the acceptable speech acts by providing common language for communication and knowledge representation.

Figure 2 System structure of the recommender system

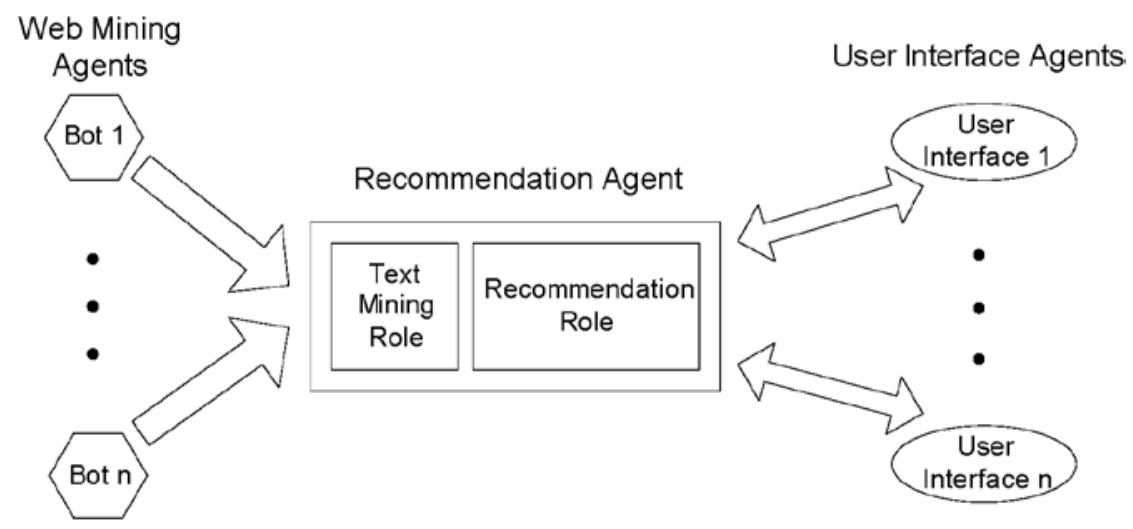

\section{Web mining agent}

Software robots, so-called bots, which are smart software tools for retrieving useful data from internet websites, can be adapt to search and categorise online product review comments in an efficient way. Bots have great potential in automating online text mining. Bots can save labour in extracting relevant texts, as they persist this in a search, and in constructing the text data sets, refining them as they go along. Sophisticated 
intelligent bots that can make decisions based on past experiences are powerful tools to perform the methodical searches to uncover information from the internet. This section presents the methods of design and implementation of smart text mining bots adopted in the multi-agent recommender system. The section includes the level of technical details, necessary for appreciating the text mining bot technology.

The first step to develop a bot is to connect to the web server that has been chosen. A number of languages can be used for the implementation of the solution, including Python, C\#, ASP, .Net and PERL, to name the few. We have chosen Java due to the convenient ontology of classes related to our problem of building large scale web crawler applications and the existing class libraries. Java socket API has been chosen for enabling the web mining agent to communicate to the web server since it is platform independent and comprehensive for network application development. Since web servers normally listen on TCP port 80 , a socket with the IP address of the website can be constructed if the IP address of the server is known. Java encapsulates the concept of an ordinary TCP socket with the class Socket, and the concept of a server socket with the class ServerSocket. Data stream (document) that is input to or output from a socket is encapsulated in Java using the InputStream and OutputStream classes, respectively. Documents on the internet web server can also be retrieved by using Java URL class. The standard identifier for a document on the internet is its Universal Resource Locator (URL). Java encapsulates the concept of a URL with the class URL.

The documents retrieved from web servers are HTML documents. The structure of the HTML document from each website is different from each one. Therefore, in general, retrieving review comments from a URL has to involve certain degree of customisation. To minimise the customisation level, an HTML document analyser package is developed to allow bots to be built on top of it. The HTML document analyser should provide the function to easily analysis the document structure and remove HTML tags in the document.

A web page normally contains not only the review comments but also other contents such as advertisements, links to other websites etc. Data extraction from web documents is usually performed by software modules called wrappers. Hard-coded wrapper by using static template is tedious, error-prone and difficult to maintain. To overcome this difficulty, significant research has been done in the area of wrapper induction, which typically applies machine learning technology to generate wrappers automatically (Chidlovskii et al., 2000; Freitag and Kushmerick, 2000). WIEN is the first wrapper induction system that defined six wrapper classes (tem-plates) to express the structures of websites (Kushmerick and Grace, 1998; Kushmerick, 2000). STALKER - a wrapper, more efficient than WIEN (Muslea et al., 1998), treats a web page as a tree-like structure and handles information extraction hierarchically. Gao and Sterling (2000) have also done significant work on knowledge-based information extraction from the internet. However, most of the earlier wrapper techniques were tailored to particular types of documents. The more recent techniques aim on data extraction from general semi-structured documents. Zhang and Simoff (2004) proposed a technique for news data identification and retrieval that takes into account the characteristics of news web pages can be employed for extracting review comments. Similar to news data, review comments are displayed on the middle of the web page and contain particular set of keywords. Without loss of generality, this approach improves the processing efficiency and requires neither user specified examples nor priori knowledge of the pages. 


\section{Recommendation agent}

Recommendation agent plays the central role in the system. It applies text mining techniques to convert the received review comments into ontology. The quality of each feature of the product is calculated using the ontology.

\subsection{Ontology mapping}

Online product reviews provide valuable source of information for recommender systems. However automating the acquisition of such information requires innovative technological solutions. The consumer reviews are textual and unstructured sources that are particularly difficult to acquire. Therefore, identifying an appropriate representation of consumer opinions that can be used in the system is a key problem. One way to convert these opinions to a structured form is to use translation ontology, which is typically used as a form of knowledge representation and sharing. An ontology is a collection of concepts and their relationships that can collectively provide an abstract view of an application domain (Gruber, 1993; Guarino et al., 1999). In this application, the ontology contains two main parts: Opinion Quality (OQ) and Product Quality, which summarise the consumer skill level and the consumer experience with the product in the review, respectively. Figure 3 shows the general structure of the ontology. The OQ includes several variables to measure the opinion provider's expertise in the product. The Product Quality represents the opinion provider's valuation of the product features, which is highly domain specific. The ontology concepts, attributes, constraints and rules were defined manually to ensure the design quality. Once the ontology is defined, it can be used until the product has new features. Protégé 3.1 was used to support the creation, visualisation, and manipulation of the ontology.

Figure 3 Structure of the ontology used in creating recommendation from consumer opinions

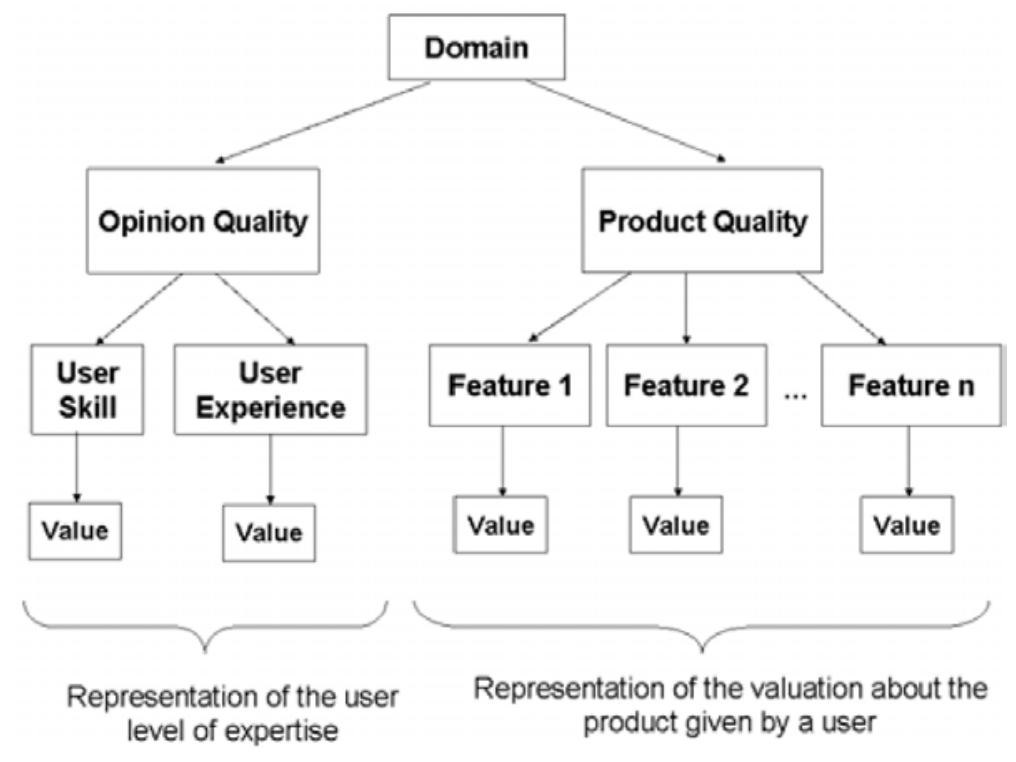


Once the ontology has been defined, a methodology is required to map review comments into ontology instances automatically. Manually mapping thousands of review comments into ontology instances is a tedious and time consuming job. Furthermore, the recommender system is built as an online service. Manual mapping is infeasible due to new review comments are added constantly. This section describes a methodology developed for the agent to create ontology instances automatically using text mining techniques. As the ontology has been defined, the mapping process includes the identification of both the classes involved in the instance and their attributes. The mapping process is composed by two steps:

- Sentence selection and classification. This step identifies the class attributes. In the user valuation from the text data, each feature from the comment is assigned either 'Good' or 'Bad'. Therefore, the sentences in the review are selected and classified into three categories: 'Good' comments, 'Bad' comments and 'Quality'. 'Quality' category contains the sentences that indicate the OQ.

- Concept identification. Once the relevant sentences are selected, this step identifies the classes that the selected sentences belong to. The concepts which implicated in the sentences determine the classes in the ontology, are identified by related words used as synonymous of the concept.

Similar to other classification applications, collection and labelling training examples for sentence classification are manual processes. Once the system has been trained, it automatically classifies a sentence into either 'Good' or 'Bad' category. In the concept identification step, the synonym database was also created manually. The concept is identified automatically if a keyword in the database is identified in the sentence.

\subsubsection{Step 1: Sentence selection and classification}

The process of sentence selection and classification is described in Figure 4. Under the text mining paradigm, each sentence is treated as a document in this application. To group review sentences into 'Good', 'Bad' and 'Quality', shallow parser was firstly considered as an analyser tool. However, most of parsers give complicate and incorrect results. Furthermore, each document is very short. Classification algorithms based on term frequencies do not provide satisfaction results either. Therefore, rule based classification techniques are employed. As described in previous section, three categories have been defined to classify the sentences: 'Quality', 'Good' and 'Bad'. 'Quality' category groups those sentences that contain information about the skill of the consumer.

'Good' category groups those sentences that contain information about some features that consumer has valuated as the strengths of the product. 'Bad' category groups those sentences that contain information about some features that the consumer considers as weaknesses of the product. At this stage, the work has been focused on providing the overall concept of utilising text mining for automatic mapping of review comments into ontology instances. Hence, we employed an off-the-shelf text mining kits. The Text-Miner Software Kit (TMSK) and the Rule Induction Kit for Text (RIKTEXT) have been used to obtain the classification rule sets (Sholom et al., 2004). TMSK generates a dictionary from a set of documents (sentences in our case) and converts a set of sentences into sparse vectors based on the dictionary. The dictionary and the vectors representing each category are used by RIKTEXT for learning 
a classifier. RIKTEXT is a complete software package for learning decision rules from document collections. The rules are induced automatically from data. The output is a rule set of classification of 'Good' 'Bad' and 'Quality' category from training data. Figure 3 shows the inputs and the output of the selection and classification process. Opinions of 68 reviews about the digital camera: Canon PowerShot SD500 (Digital IXUS 700) from www.dpreview.com web have been used to create the training data set. Each sentence of each review is treated as a document. 195 sentences have been obtained for the 'Good' category, 127 sentences for 'Bad' category and 47 sentences for 'Quality' category. The available data have been spited into training and tests portions. Test cases are selected randomly in RIKTEXT and we specified how many cases should be used for testing. We choose two-thirds of the available cases for training and the rest for testing.

Figure 4 Inputs and outputs for classifier consumer's re-views process
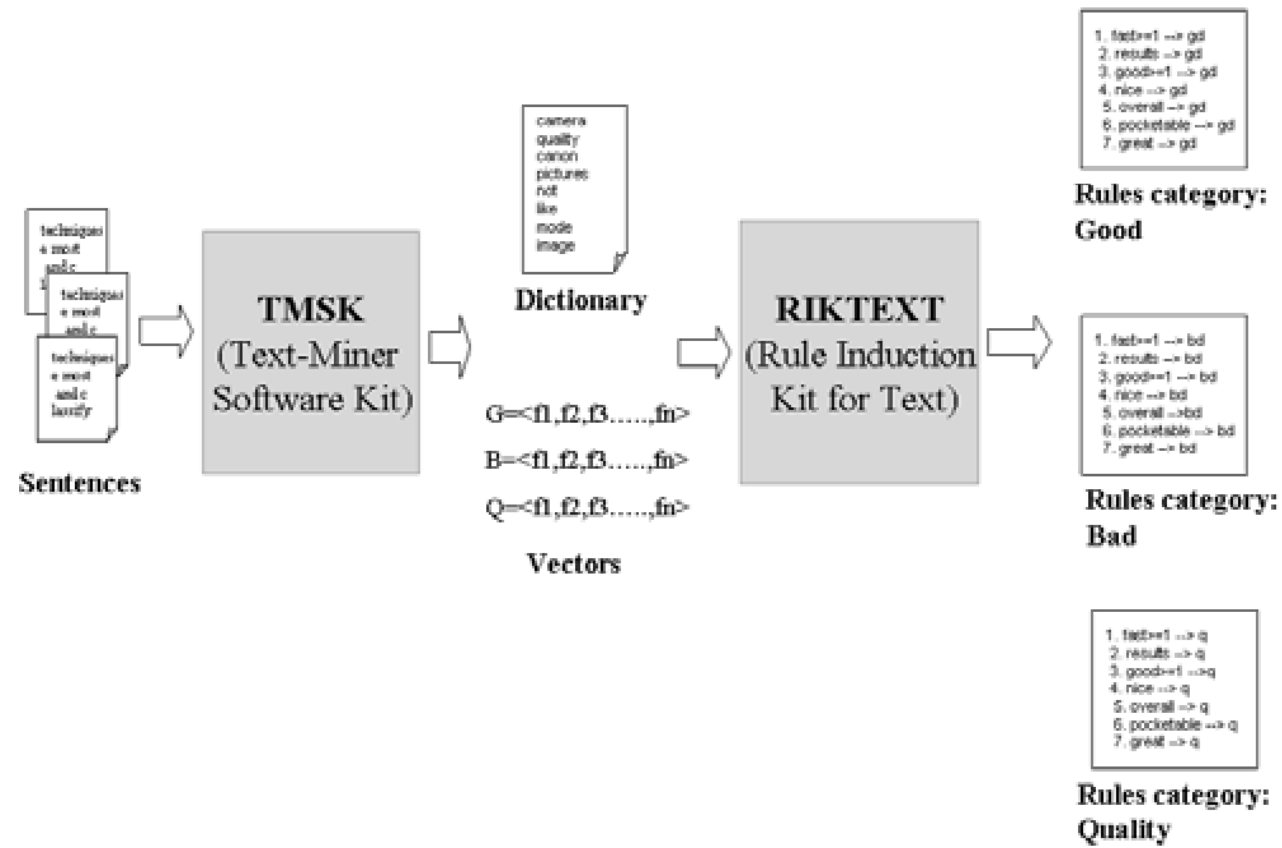

\subsubsection{Step 2: Concept identification}

Once the sentences have been classified into one of the categories, the concept (class) in the ontology implicated in the sentence is needed to be identified. Each concept in the ontology contains a label name and a related word list. A related word list of a concept contains vocabulary (a set of keywords) through which the concept can be matched with one sentence in the comments. An example of the synonym database is shown in Figure 5. 
Figure 5 Synonym database for concept identification

\begin{tabular}{|ll|}
\hline FEATURES & \\
& SYNONYMOUS \\
auto_setting & autosetting \\
battery & battery \\
button & butoons \\
button & button \\
camera & camera \\
camera & feature \\
camera & features \\
camera & functions \\
card & card \\
card & cf-card \\
card & sd \\
card & sd-card \\
color_balance & color balance \\
color balance & colour balance \\
color_balance & consistent balance \\
color_balance & white balance \\
comparison & compare \\
comparison & compared \\
comparison & comparison \\
comparison & equal \\
comparison & same \\
…… & \\
\hline
\end{tabular}

\subsection{Product rating calculations}

The product rating is calculated by the consumer skill level and product quality ranking using the data in the ontology instances obtained from the previous step.

\subsubsection{Rating the consumer skill level}

The review comments were given by people with diverse experience and skill levels. In general, people who have longer history of using the product can provide more professional opinions. Therefore, these diverse opinions should not be treated equally. The opinions from more experienced people should be taken in account to a greater extent than those from people with little knowledge of the product. OQ is defined to evaluate the weighting value of opinions according to the opinion providers' expertise.

Definition 1: OQ is the sum of the weight $\mathrm{w}_{\mathrm{j}}, \mathrm{j}$ given for each variable representing the skills and experiences of consumer i divided by the number of variables representing the information about consumer's skill and expertise provided in the ontology.

$$
\mathrm{OQ}_{\mathrm{i}}=\frac{\sum_{\mathrm{j}}^{\mathrm{n}} \mathrm{w}_{\mathrm{j}}}{\mathrm{n}} .
$$

The OQ is calculated by the values stored in the corresponding part of the ontology. Detail definition of the variables is given in Section 3.2. An OQ value is calculated for each piece of comment. 


\subsubsection{Product quality ranking}

The product is ranked according to the consumer comments for each feature. Due to the difficulties of quantification of user valuation from texture data, each feature from the comment can only be assigned either 'Good' or 'Bad', which is calculated as ' 1 ' or ' -1 ' respectively.

For each feature, a Feature Quality (FQ) is calculated, which is a function of consumer valuation and $\mathrm{OQ}$.

Definition 2 (FQ): The quality value for each feature of the product in a review is the rating multiply by the $\mathrm{OQ}$ value of the consumer

$$
\mathrm{FQ}_{\mathrm{f}}=\mathrm{r} \times \mathrm{OQ}_{\mathrm{i}} .
$$

For the convenience of recommendation, the Overall Feature Quality (OFQ) of each feature of each product is calculated from related comments and stored in the database.

Definition 3: OFQ is the global valuation of the feature from all reviews, which is calculated by the average value of $\mathrm{FQ}$.

$$
\mathrm{OFQ}_{\mathrm{f}}=\sum_{\text {Number of Opinions }}^{\sum(\text { Scaling Factor } \times \mathrm{FQ})} .
$$

In this equation, Scaling Factor is used for the minor adjustment of the user valuation, which can be set to:

$$
\text { Scaling Factor }=\frac{1}{\mathrm{n}}
$$

$\mathrm{n}$ is the number of all the features rated by the consumer. Each review rated different number of features so $n$ could be different.

\section{User interface agent}

User interface agents are used for managing the input/output aspects of the system. They provide personalised assistance to users with their requirements. A user interaction profile that enables the interface agent to enhance and personalise their interaction with users can be defined and stored in the system (Schiaffino and Amandi, 2004).

For our system, let a user request "I would like to know if Sony361 is a good camera, specifically its interface and battery consumption” be presented. Three keywords defined in the synonym database (Sony361, interface and battery) are firstly identified. The user interface agent requests the opinions of interface and battery for Sony361 from the recommendation agent. The recommendation agent retrieves the OFQ of these two features of the requested camera from the database and calculates an Overall Assessment (OA) score by these two values: 
Definition 4: OA provides a final score of the product based on the valuation of each feature. It is calculated as the sum of all OFQ (calculated by equation (3)) multiplied by the Importance Index.

$$
\mathrm{OA}=\sum \mathrm{OFQ} \times \text { Importance Index. }
$$

The Importance Index measures the different influence of the features to consumer's decision making, which can be assigned in two ways: according to the importance of the feature expressed in the user request or by the frequency that the features have been rated in the consumer reviews.

The recommendation agent also calculates the OA of all brands and provides a suggestion of the brand that has the highest value. Figure 6 shows the output of the user request.

To avoid users to request recommendations of the products and features those are not within the capability of the system, the user interface uses combo boxes to let user to choose the brand and model of the products, as shown in Figure 7.

Figure 6 User interface agent: presenting recommendations, based on consumers’ opinions in response to a human request

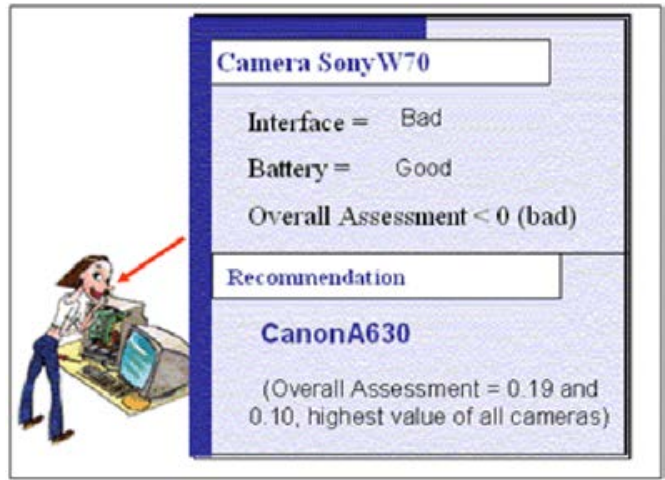

Figure 7 User interface agent: taking an input from the user

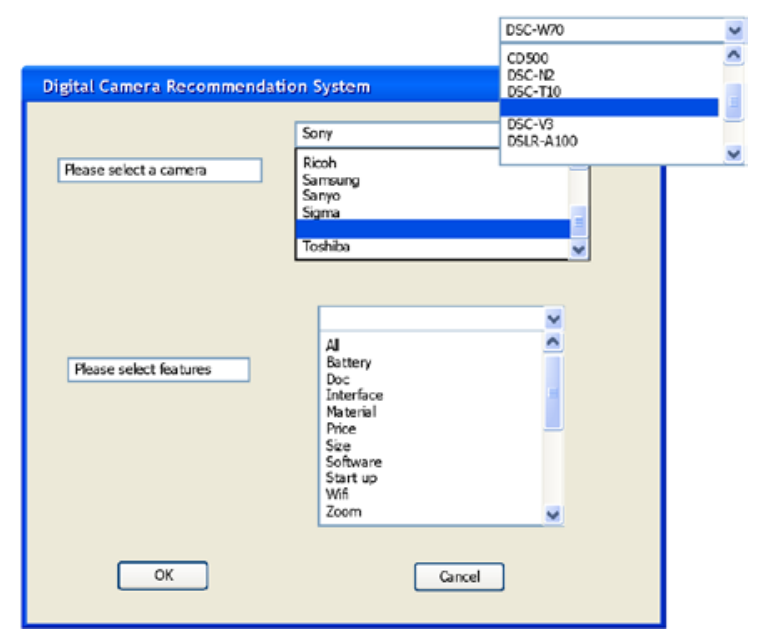




\section{Notes on implementation}

As the agent communication is supported by electronic institution, we only need to define the communication protocol between agents. For this system, there are mainly two types of communications:

- between web mining agents and recommendation agent

- between recommendation agent and user interface agents.

Web mining agents inform recommendation agent when new data (review comments) is available. At the end of each day, the recommendation agent checks if there is any new data arrives. It processes the review comments into the review comment ontology which are expressed as XML files. After finish processing all the comments, it updates the OFQ values for the affected products.

The recommendation agent is designed as a multithreaded agent. It has a listener for handling incoming connection requests from user interface agents. It processes the connection if the electronic institution recognises the agent and permits it to enter the system. A request and response ontology is defined to assist the communication between these two agents.

\section{Conclusions}

This paper presents an implementation of a multi agent recommender system that bases on consumer review comments. A ranking mechanism for prioritising the product quality with respect to the consumer level of expertise and the rating given to some features of the product has been developed. The approach uses domain ontology to translate the information into a form that is suitable for processing by the recommender system. Such ontology has been defined for the domain of digital camera reviews and has been used for demonstration of the work with some examples. A set of measures such as $O Q$, $\mathrm{FQ}, \mathrm{OFQ}$ and OA have been defined to select the relevant reviews and provide the best recommendation in response to a user request. The recommendation is given based on these measurements.

In the future work, the implemented system should be evaluated with the intended consumer groups in a real-world application.

\section{References}

Aciar, S., Zhang, D., Simoff, S.J. and Debenham, J.K. (2007) 'Informed recommender: basing recommendations on consumer product reviews', IEEE Intelligent Systems, Special Issue on Recommender Systems, May-June, pp.39-47.

Adomavicius, G. (2005) 'Toward the next generation of recommender systems: a survey of the state-of-the-art and possible extensions', IEEE Transactions on Knowledge and Data Engineering, Vol. 17, No. 6, pp.734-749.

Chevalier, J. and Mayzlin, D. (2003) The Effect of Word of Mouth on Sales: Online Book Reviews, NBER Working Paper Series, National Bureau Of Economic Research, USA.

Chidlovskii, B., Ragetli, J., and de Rijke, M. (2000) 'Automatic wrapper generation for web search engines', Proceedings of the 1st International Conference on Web-Age Information Management WAIM’00, LNCS 1846, Springer, Heidelberg, pp.399-410. 
Curien, N., Fauchart, E., Laffond, G. and Moreau, F. (2006) 'Online consumer communities: escaping the tragedy of the digital commons', in Brousseau, E. and Curien, N. (Eds.): Internet and Digital Economics, Cambridge University Press, Cambridge, pp.201-219.

Dellarocas, C. (2003) 'The digitization of word-of-mouth: promise and challenges of online feedback mechanisms’, Management Science, Vol. 49, No. 10, pp.1407-1424.

Dellarocas, C. (2004) Strategic Manipulation of Internet Opinion Forums: Implications for Consumers and Firms, Working Paper, Sloan School of Management, MIT, Cambridge (Aciar et al., 2006).

Freitag, D. and Kushmerick, N. (2000) 'Boosted wrapper induction', Proceedings of the 17th National Conference on Artificial Intelligence, AAAI-2000, AAAI Press/MIT Press, Boston, Massachusetts, pp.577-583.

Gao, X. and Sterling, L. (2000) 'Semi-structured data extraction from heterogeneous sources', in Schwartz, D.G., Divitini, M. and Brasethvik, T. (Eds.): Internet-based Knowledge Management and Organizational Memories, Idea Group Publishing, Hershey, USA, pp.83102.

Gruber, T.R. (1993) 'A translation approach to portable ontology specifications', Knowledge Acquisition, Vol. 5, No. 2, pp.199-220.

Guarino, N., Masolo, C. and Vetere, G. (1999) 'OntoSeek: content-based access to the web', IEEE Intelligent Systems, Vol. 14, No. 3, pp.70-80.

Kushmerick, N. (2000) 'Wrapper induction: efficiency and expressiveness', Artificial Intelligence, Vol. 118, Nos. 1-2, pp.15-68.

Kushmerick, N. and Grace, B. (1998) 'The wrapper induction environment', in Baxter, J. and Logan, B. (Eds.): Software Tools for Developing Agents: Papers from the AAAI Workshop, 94-100, Technical Report WS-98-10. American Association for Artificial Intelligence, Menlo Park, California, pp.131, 132.

Muslea, I., Minton, S. and Knoblock, C. (1998) 'STALKER: learning extraction rules for semistructured, web-based information sources', AI and Information Integration: Papers from the AAAI Workshop, 94-100, Technical Report WS-98-14. American Association for Artificial Intelligence, Menlo Park, California, pp.74-81.

Schiaffino, S. and Amandi, A. (2004) 'User - interface agent interaction: personalization issues', Int. J. Human-Computer Studies, Vol. 60, No. 1, January, pp.129-148.

Senecal, S. and Nantel, J. (2004) 'The influence of online product recommendations on consumers' online choices’, Journal of Retailing, Elsevier, Vol. 80, pp.159-169.

Sholom, M.W., Indurkhya, N., Zhang, T. and Damerau, F. (2004) Text Mining: Predictive Methods for Analyzing Unstructured Information, Springer-Verlag, New York.

Sierra, C., Rodríguez-Aguilar, J.A., Noriega, P., Esteva, M. and Arcos, J.L. (2004) 'Engineering multi-agent systems as electronic institutions', European Journal for the Informatics Professional, Vol. 4, pp.33-39.

Zhang, D. and Simoff, S. (2004) 'Informing the curious negotiator: automatic news extraction from the internet', in Simoff, S.J. and Williams, G.J. (Eds.): Proceedings of the 2nd Australasian Data Mining Conference, Cairns, Australia, 6-7 December, pp.55-72. 